\title{
La inserción laboral de los pedagogos en el marco de las políticas activas de empleo
}

\section{A inserção laboral de pedagogos no marco das políticas ativas de emprego}

\section{The labour insertion of the pedagogues in the framework of the active labour market policies}

\author{
Miguel A. Santos Rego* \\ Jesús García Álvarez*
}

\begin{abstract}
RESUMEN
Las actuales políticas activas de empleo constituyen uno de los pilares fundamentales del funcionamiento del mercado de trabajo, contribuyendo a la creación de trabajo y facilitando la inserción de los desempleados, entre ellos, los graduados universitarios, uno de los colectivos de atención prioritaria. Por ello, nos hemos propuesto realizar una revisión del impacto que las actuales políticas activas tienen en su inserción laboral, a través de un estudio realizado en la Universidad de Santiago de Compostela (España) con el alumnado próximo a graduarse en pedagogía. Hemos usado una metodología básicamente descriptiva a partir de un cuestionario sobre la inserción laboral de estos graduados (muestra de 169 sujetos). Concluimos afirmando la necesidad de impulsar estas políticas en el escenario de la educación superior, en tanto que se han demostrado las más adecuadas en términos de empleabilidad. Igualmente, defendemos la realización de evaluaciones de impacto, sobre todo, centradas en los resultados de tales políticas y posibles vías de mejora.
\end{abstract}

Palabras clave: Políticas activas de empleo. Educación superior. Graduados en pedagogía. Inserción laboral.

DOI: $10.1590 / 0104-4060.49146$

* Universidad de Santiago de Compostela. Facultad de Ciencias de la Educación. Santiago de Compostela, Galiza, Espanha. Rúa Xosé María Suárez Núñez, CP: 15782. E-mail: miguelangel. santos@usc.es y jesus.garcia.alvarez@usc.es. 


\title{
RESUMO
}

As atuais políticas ativas de emprego constituem um dos pilares fundamentais do funcionamento do mercado de trabalho, contribuindo para a criação de empregos e facilitando a inserção dos desempregados, entre eles, os graduados universitários, um dos coletivos de atenção prioritária. Portanto nos propusemos a realizar uma revisão do impacto que as atuais políticas ativas de emprego têm em sua inserção laboral por meio de um estudo realizado na Universidade de Santiago de Compostela (Espanha) com o alunado próximo a se graduar na titulação de Pedagogia. Usamos uma metodologia basicamente descritiva por meio de um questionário sobre o tema da inserção laboral destes graduados (amostra de 169 sujeitos). Concluímos ao afirmar a necessidade de impulsionar essas políticas no cenário da educação superior, uma vez que elas têm demostrado serem as mais adequadas em termos de empregabilidade. Igualmente, defendemos a realização de avaliações de impacto, sobretudo, centradas nos resultados de tais políticas e possíveis vias de melhora.

Palavras-chave: Políticas ativas de emprego. Educação superior. Graduados em pedagogia. Inserção laboral.

\begin{abstract}
The current labour policies are one of the essential pillars in the operation of the job market, contributing to job creation and facilitating the insertion of the unemployed, including graduates as a group that merits priority attention. Thus, the aim of this study is to conduct a review of the impact that current active labour policies have on the graduate's employability, through a research with students in the last year of their degree in Pedagogy, implemented at the University of Santiago de Compostela (Spain). We used descriptive methodology, applying a questionnaire on the topic of the graduate's insertion into the job market (sample of 169 subjects). We conclude stating the necessity to promote these policies in the scenario of higher education, as they are the most appropriate in terms of employability. Similarly, we support the carrying out of impact assessments mainly focused on the results of such policies and on the possible ways to improve them.
\end{abstract}

Keywords: Active labour policies. Higher education. Graduates in pedagogy. Insertion into the job market. 


\section{Introducción}

La adaptación del sistema universitario español al Espacio Europeo de Educación Superior (EEES) supuso la redefinición, a escala global, de la oferta universitaria existente. Con la promulgación de los Reales Decretos de estudios oficiales de Grado ${ }^{1}$ y Posgrado ${ }^{2}$ se transformó la estructura universitaria y, con ello, la responsabilidad de reorganizar las titulaciones.

Esta normativa ofrecía un marco jurídico, que posibilitaba a las universidades la organización -con flexibilidad y autonomía- de las enseñanzas oficiales a fin de lograr su armonización con las establecidas desde el ámbito estatal. Sin embargo, pasar de un sistema con una determinada estructura, que, como en el caso de España es de difícil comparación con el resto de Europa, a otro requería de la colaboración efectiva de diversos organismos públicos y de su compromiso ante el reto. Para ello, la Agencia Nacional de Evaluación de la Calidad y Acreditación (ANECA), de acuerdo con el proceso de convergencia, activó a universidades, asociaciones y colegios profesionales para que facilitaran, previa elaboración de un informe marco, la adaptación y posterior implementación de las titulaciones al nuevo contexto europeo. Desde luego, la creación de una Área Europea de Educación Superior supuso el establecimiento de un conjunto de principios clave:

- Adopción de una tipología de titulaciones fácilmente comprensible y comparable.

- Establecimiento de un sistema de créditos para facilitar la movilidad de estudiantes.

- Promoción de la movilidad para el ejercicio efectivo del libre intercambio (alumnado, profesores e investigadores, entre otros).

- Promoción de la cooperación europea con la finalidad de garantizar la calidad a través del desarrollo de criterios y metodologías comparables.

- Promoción de las dimensiones europeas necesarias en educación superior, dirigidas al desarrollo curricular, cooperación entre instituciones y esquemas de movilidad y programas de estudio.

Esto requería, en un primer momento, identificar con claridad el desarrollo de las diferentes fases para poner en marcha el nuevo modelo de educación superior y, a su vez, el establecimiento de las bases para la consecución de sus

1 Real Decreto 55/2005, del 21 de enero, por el que se establece la estructura de las enseñanzas universitarias y se regulan los estudios universitarios oficiales de Grado (BOE, 25/01/2005).

2 Real Decreto 56/2005, del 21 de enero, por el que se regulan los estudios universitarios oficiales de Posgrado (BOE, 25/01/2005). 
objetivos. Con la Declaración de Bolonia (1999), en el año 2000, un grupo de universidades aceptaba colectivamente el reto propuesto y elaboraba un proyecto piloto denominado Tuning-Sintonizar las estructuras de Europa. Este proyecto tenía en cuenta la declaración, pero, como no podía ser de otro modo, se preocupaba por la adopción de una estructura de titulaciones fácilmente reconocibles y comparables, de un modelo basado en dos ciclos y del establecimiento de un sistema de créditos. Se iniciaba, así, la entrada en un proceso de cambio educativo en los países miembros, con el propósito de crear un modelo de fácil comprensión, que promoviera oportunidades de trabajo para los estudiantes y una competitividad mucho mayor en el ámbito europeo. Asumidos los objetivos de la declaración y con un nuevo diseño de la arquitectura académica de las titulaciones, el Ministerio de Educación diseña el documento marco La integración del sistema educativo español en el Espacio Europeo de Enseñanza Superior que se constituiría como punto de referencia y guía base para la integración en el EEES.

En la actualidad, el proyecto ya constituido, a partir de lo que antes eran los estudios de licenciatura y ahora son los de grado, revela sus principales consecuencias en la formación de los graduados. Desde tales coordenadas, es oportuno conocer si realmente este nuevo modelo responde, no solo a las exigencias y necesidades del actual marco socio-laboral, sino también a las expectativas de los propios graduados. El marco socio-laboral en el que ha de proyectarse su dinámica profesionalizadora se caracteriza por la aparición de cambios en rápida sucesión, que afectan las condiciones de empleabilidad -coyuntura económica, ofertas de empleo y demandas de trabajo- y de ocupabilidad -actitudes, competencias o factores sociales- (ROMERO; PÉREZ; VIDAL; JUEZ, 2004). Esto produce desconocimiento sobre la configuración de su contexto profesional de referencia, incertidumbre ante las nuevas situaciones laborales, escasa congruencia entre la formación adquirida y la exigida por los empleadores, aparición de nuevas pautas de interacción con el mercado en la búsqueda de empleo, exiguo conocimiento de los factores y recursos necesarios en términos de inserción laboral, readaptaciones continuas en los contenidos de cada titulación y modificación de expectativas y aspiraciones académico-laborales (ANECA, 2004). Surge, así, un debate asociado al proceso de inserción de los universitarios en relación a las posibilidades y oportunidades que hacen posible su acceso al empleo.

Justamente, por ello, es importante dar cuenta de las expectativas laborales del alumnado que finaliza sus estudios, ya que condicionan su situación y constituyen un elemento de reflexión ante la compleja transición de la esfera académica al mercado de trabajo. No se puede olvidar que la inserción de los titulados al mundo laboral es una de las mayores finalidades de la educación 
superior, lo cual afecta la evaluación de los resultados. Las universidades son los principales núcleos de formación de los recursos humanos, por lo que se vuelve necesario una colaboración constante y posibilitadora de nuevos modelos formativos, a la vez que profesionalizadores, susceptibles de favorecer la inserción laboral de las nuevas generaciones (CAJIDE, 2004).

Es imprescindible que la educación superior se diversifique y se ajuste a las nuevas necesidades sociales, colaborando con los demás sistemas a fin de proporcionar las competencias, capacidades y habilidades necesarias para afrontar una posible inserción laboral (SANTOS REGO; REQUEJO OSORIO; RODRÍGUEZ MARTÍNEZ, 1993). En este sentido, destaca la constante preocupación en relación con los retos y demandas sociales, educativas, científicas y tecnológicas, pues lo que interesa es revisar y redefinir sus políticas de planificación, de administración y de gestión, readaptando sus funciones, perfiles profesionales y servicios universitarios (TÜNNERMANN, 2000). Tales medidas desempeñan una función fundamental en los sistemas de educación superior, donde la pedagogía académica juega un papel determinante con la finalidad de incentivar secuencias de desarrollo que vinculen teoría y praxis de la empleabilidad. Es, sin duda, una cuestión de buena educación, no solo de buena actuación ante objetivos mercantiles, legítimos pero insuficientes ante la presión desmedida por influir en el gobierno de las universidades (SANTOS REGO, 2014).

Si son analizadas las transformaciones de las instituciones que intervienen en el proceso de transición de los jóvenes al mundo laboral, se advierte la influencia de cuatro factores: el cambio en la estructura de empleo y la naturaleza de las calificaciones requeridas, el alargamiento de la formación inicial, la transformación de las estrategias empresariales en la selección de personal y la intervención de los poderes públicos para establecer mecanismos especiales de inserción laboral (CACHÓN, 2004). Este último factor es el que interesa. Los poderes públicos se encargan de gestionar y sistematizar la política de empleo en programas concretos, cuya finalidad no es otra que facilitar la inserción en el mercado laboral o, en su defecto, la proporción de herramientas que la hagan efectiva. Y es a esto a lo que se llama políticas activas de empleo (JOVER, 2006).

El presente estudio, centrado en la inserción socioprofesional de los titulados universitarios, especialmente de los que han estudiado pedagogía, pretende contribuir a la gestión del cambio en los mismos contextos de formación, pero partiendo del análisis de las principales políticas que promueven el acceso al empleo. Serán atendidas las variables que intervienen de modo directo en esta dinámica y se abordará, por un lado, las posibilidades de inserción y, por otro, las actuales medidas de formación y orientación laboral. 


\section{El papel de las políticas activas de empleo en la inserción laboral de los titulados universitarios}

Al referirse a los principios sociales y económicos del Estado democrático de derecho, la Constitución Española (artículos 40 y 41) establece el compromiso de la administración pública con el desarrollo de una política orientada hacia el pleno empleo, que asegure la garantía de la formación, la readaptación profesional y la protección en caso de desempleo (SÁIZ, 2004). En este marco emergen las políticas activas, a las que la Ley de Empleo ${ }^{3}$ dedica un capítulo específico, definiéndolas como el conjunto de decisiones que contribuyen al desarrollo de programas y medidas proclives a la consecución y mejora de la calidad en el trabajo, la adecuación cuantitativa y cualitativa de la oferta y la demanda, la reducción del paro, y la debida protección en las situaciones de inestabilidad laboral (SALVÀ, 2009).

Sin embargo, es necesario que tales políticas se acoplen al sistema de protección estatal y se desarrollen teniendo en cuenta la Estrategia Europea de Empleo $2020^{4}$ y las necesidades de los demandantes, además de los requerimientos de los respectivos mercados de trabajo. Para la consecución de tal fin, los objetivos establecidos pasan por informar y orientar hacia la búsqueda activa de empleo, desarrollar programas de formación profesional ocupacional y continua, facilitar la práctica profesional, crear y fomentar el empleo estable y de calidad, promover la creación de actividad que genere ocupación, facilitar la movilidad geográfica y promover políticas destinadas a la inserción laboral de personas en situación de riesgo o de exclusión (BARBA, 2012). Todo ello supervisado, de manera coordinada, entre los agentes de formación profesional e intermediación laboral.

En este marco, la OCDE (2012) ha puesto de manifiesto la influencia que las políticas activas de empleo ejercen sobre el mercado de trabajo, produciendo un aumento en el nivel de empleo o reducción en el de desempleo. Esto constituye lo que se conoce como Derecho al Empleo, al ser traducido a sus correspondientes normas jurídicas. Por lo tanto, esa política, en relación al conjunto de decisiones de los poderes públicos, se configura en un plano normativo, donde se sitúa el Derecho al Empleo, encargado de ordenar de forma

3 Ley 56/2003, de 16 de diciembre, de Empleo (BOE, 17/12/2003).

4 La Estrategia Europea de Empleo 2020 constituye un conjunto de orientaciones en las que se establece la elaboración de planes nacionales con el objetivo de crear más puestos de trabajo de calidad en toda la Unión Europea (UE). 
institucional y jurídica la oferta y la demanda, siempre desde las orientaciones políticas adecuadas (FERNÁNDEZ DOCAMPO, 2013).

Asimismo, la repercusión de estas medidas ha evolucionado en relación a las distintas corrientes teóricas de cada etapa, del modelo económico asumido o de las prioridades políticas de cada gobierno. De hecho, ya en 1994, el informe Job’s Study, referencia básica en materia de estrategias para la reducción del desempleo, reconocía las políticas activas como un recurso esencial en la mejora del funcionamiento del mercado de trabajo, siempre y cuando fuesen asumidas con carácter complementario a otras medidas (OCDE, 1994). No obstante, se observa que, en relación a otras acciones, las políticas activas desempeñan un papel relevante en la articulación del mercado de trabajo, sobre todo en lo que se refiere al empleo de los jóvenes. Y es que, el futuro de las nuevas generaciones nunca se ha visto tan comprometido por una situación de crisis económica global como la que se atraviesa en la actualidad. Los datos de las instancias internacionales sobre los niveles de desempleo juvenil no son alentadores precisamente; e incluso pueden llevarnos a la representación de una realidad con predominio de la desorientación, la precariedad, la falta de oportunidades de trabajo digno, la desmotivación y un elevado nivel de abandono temprano de los estudios (HERNÁNDEZ-BEJARANO, 2013).

\section{La inserción laboral de los titulados superiores en el marco de las políticas activas de empleo y el caso de los graduados en pedagogía}

Es indudable que los recientes títulos de grado fueron creados con la pretensión de mejorar la calidad de la educación superior, de diversificarla y de adaptarla de un modo más práctico a los nuevos entornos de trabajo. Ante este escenario, las universidades han asumido la responsabilidad en materia de empleabilidad, dando a conocer cómo, cuándo y en qué condiciones se produce el acceso al mercado laboral, lo que, indefectiblemente, exige información sobre los titulados. En Galicia (España), la Agencia para la Calidad del Sistema Universitario de Galicia (ACSUG, 2014), en su estudio sobre la inserción laboral de los graduados, vuelve sobre la cuestión al afirmar que una de las labores fundamentales de las universidades es preparar a los universitarios para el acceso al mercado laboral, el cual es competitivo, ya no tiene fronteras y está en constante cambio.

Lo que se presenta aquí es un estudio de naturaleza no experimental, construido fundamentalmente a partir de escalas previamente validadas. Para 
la recolección de datos se elaboró un cuestionario compuesto por 67 ítems, agrupados en diferentes bloques. A nivel general, se incluyeron factores de carácter personal, académico, de la titulación cursada y de la situación laboral actual, entre otros, de modo tal que se pudiera extraer una serie de variables que fueran medibles con facilidad. Se tuvieron en cuenta cuestionarios como el elaborado por la ANECA en su estudio sobre la inserción laboral de los titulados en España (influenciado por el utilizado en el proyecto internacional CHEERS $^{5}$ ) y cuestionarios como el de la ACSUG, sobre la inserción laboral de los titulados gallegos.

La investigación se planteó con carácter longitudinal y los datos hacen referencia a la valoración de tres de las promociones de último curso del grado en pedagogía de la Universidad de Santiago de Compostela (2013-2014, 2014-2015 y 2015-2016). Partimos de una población estimada (N) de 209 estudiantes, con una muestra productora de datos de 169 sujetos. Se encuentran, en su mayoría, en un rango de edad de 21 a 23 años (77.2\%). La pedagogía constituye para el $82.6 \%$ un estudio de primera o segunda opción y, al igual que en otros estudios, se da una presencia mayoritaria del colectivo de mujeres $(87 \%)$, en línea con una dinámica de creciente feminización de la educación superior.

Por su parte, la variedad y cantidad de datos obtenidos a través de la aplicación del cuestionario requirió del empleo de herramientas de carácter informático. Concretamente, el paquete estadístico SPSS (versión 20) permitió describir y etiquetar las variables y sus correspondientes valores, estableciendo porcentajes y estimaciones sobre los resultados obtenidos.

En particular, los titulados en pedagogía son profesionales que, en cuanto a formación, orientación e inserción, han de sentirse preparados, aunque los datos no compadecen con tal premisa. Estos estudiantes se sienten poco motivados para emprender en el terreno laboral y sus expectativas se ven afectadas por la disociación percibida entre titulación y demandas de trabajo. La mayor parte de los encuestados no encuentra vínculos estables entre su formación y el mercado laboral; es más, tampoco creen que los contenidos o las competencias generales y específicas de la titulación estén favoreciendo su acceso al empleo. Así, los graduados emiten valoraciones negativas acerca de su formación, profesionalización e inserción, que derivan de una propuesta académica inconexa con su contexto laboral de referencia.

El presente estudio analiza elementos que han resultado fundamentales en la dinámica laboral de cualquier titulado superior. En la percepción de los

5 Proyecto CHEERS (Career after Higher Education: a European. Research Study). Estudio de investigación sobre el desarrollo profesional una vez concluidos los estudios superiores, destacando las competencias que se demandan a los estudiantes ya titulados. 
graduados de dichos elementos han de pivotar puntos de reflexión por parte de las instancias públicas. En cuanto a su preparación, la mayoría destaca la necesidad de completar la formación recibida (98.2\%), concordando con la investigación realizada por Ruiz de Miguel y García de la Barrera Trujillo (2013), en la cual el $89.5 \%$ de los sujetos manifestaba haber participado en alguna actividad formativa para completar sus estudios, lo cual habría influenciado en su acceso al empleo.

También es alto el porcentaje de alumnos (66.5\%) que no encuentra un vínculo entre su formación académica y la realidad sociolaboral, hasta el punto de que todas y todos los encuestados (100\%) ven necesario el incremento de las actuales medidas de formación y orientación laboral de las que pueden hacer uso. El nuevo marco normativo para coordinar y ejecutar estas medidas es la Estrategia Española de Empleo 2012-2014. Esta estrategia alude a seis objetivos, que han de conseguirse a través de la política de empleo: 1) elevar la tasa de empleo al 74\% para la población entre 20-64 años y la tasa de empleo femenina al $68.5 \%$; 2) reducir la temporalidad y la segmentación laboral; 3) reforzar el trabajo a tiempo parcial y la flexibilidad interna; 4) mejorar y adecuar las competencias profesionales a la demanda del mercado; 5) promover la rápida y adecuada reinserción de las personas en el mercado de trabajo; y 6) promover la igualdad de género en el ámbito laboral.

Al mismo tiempo reconoce a los jóvenes como eje prioritario de las políticas activas de empleo y, en especial, a aquellos con dificultades de inserción laboral. Así, este grupo, aparece de forma expresa en seis de los ámbitos de actuación: orientación profesional, oportunidades de empleo y fomento de la contratación, oportunidades de empleo y formación, fomento de la igualdad de oportunidades en el empleo, autoempleo y creación de empresas, promoción del desarrollo y actividad económica territorial. Y lo hace de forma implícita en los tres ámbitos restantes: ámbitos de formación y recualificación, oportunidades para colectivos especiales y fomento de la movilidad.

De esta manera se detectan diferencias entre las acciones destinadas a los jóvenes en función de la edad y el nivel formativo alcanzado. Se debe tener en cuenta que lo que distingue a estas políticas es su capacidad de ejercer simultáneamente una influencia positiva sobre las personas y el mercado laboral al pretender una mayor igualdad de oportunidades en el acceso al trabajo, ejerciendo un impacto positivo sobre aquellas que se encuentran temporalmente en paro (COMISIÓN EUROPEA, 2010).

En la esfera universitaria, los sistemas e instituciones de educación superior han experimentado una serie de transformaciones de orden estructural en relación a los procesos de crecimiento, diversificación y especialización (RODRÍGUEZ GÓMEZ, 2002). Estas reformas modificaron no solo la organización, gobierno y gestión, sino que establecieron nuevos esquemas de transferencia de fondos 
y renovaron las relaciones entre el Estado, las instituciones y la sociedad en general (GOEDEGEBUURE et al., 1994). Se entiende, pues, el compromiso de las administraciones públicas para que los titulados superiores puedan beneficiarse de algunas medidas y programas existentes, intentando mejorar su posterior desempeño profesional (ACUÑA, 2012).

No obstante, las expectativas de los actuales estudiantes son prácticamente bajas. En lo que se refiere a la percepción de acceso a un empleo, el 71.5\% expresa sentirse poco formado para enfrentarse al contexto laboral de su área profesional, y solo un $44.3 \%$ manifiesta conocer las salidas ligadas a la pedagogía. Además, las expectativas asociadas a una potencial inserción laboral se ven afectadas por las perspectivas de futuro, motivación para el éxito, necesidad de orientación profesional y posibilidades de acceso al mercado. Y son las políticas activas de empleo las que inciden en el aumento de estas posibilidades, pues entre sus propósitos está el conseguir una inserción temprana, en un contexto en el que sea posible compaginar estudios y empleo, reduciendo, con ello, las dificultades de acceso al mercado de trabajo (ALONSO; FERNÁNDEZ RODRÍGUEZ; NYSSEN, 2008).

Por otra parte, de acuerdo con la Organización Internacional del Trabajo (OIT), cerca de 75 millones de jóvenes en todo el mundo están desempleados, lo que equivale a decir que desde el año 2007 existirían, al menos, 4 millones más de jóvenes en tal situación. Es en este colectivo se aprecian nítidamente los efectos de la crisis sin que se observen mejoras inequívocas en las economías desarrolladas (OIT, 2012), especialmente en los Estados miembros de la UE. Esta situación se agrava en el contexto español por las elevadas tasas de paro entre los recién graduados. Es así que interesaría conocer qué medidas se han pensado para estas personas, sobre todo teniendo en cuenta la eficacia limitada demostrada hasta ahora por los Servicios Públicos Estatales y los Centros de Orientación e Información de Empleo (COIE), creados en algunas Universidades y concebidos como centros asesores y gestores para la inserción laboral de universitarios. Pese a ello, una investigación realizada en el panorama europeo, sitúa como medidas ineludibles para la optimización del proceso de inserción: el apoyo a los jóvenes a través de la orientación personalizada, la información sobre las oportunidades laborales y el desarrollo de programas estructurados que impulsen la empleabilidad (CALIENDO; SCHMIDL, 2016), permitiendo a los servicios de orientación universitarios establecer líneas claras de actuación.

Al mismo tiempo, en un estudio realizado por el Instituto Nacional de Empleo (2000), en el que participaron 3.336 universitarios, se apreciaron las evidencias del impacto positivo en aquellos titulados que se habían beneficiado de las acciones o programas en el marco de las políticas activas, aun cuando estas se han modificado y reducido a pequeñas líneas de actuación dirigidas a 
colectividades muy específicas (ROCHA, 2010); e incluso se ha demostrado que supone el principal elemento de mejora de la estabilidad laboral para muchos jóvenes (MINISTERIO DE EMPLEO Y SEGURIDAD SOCIAL, 2013).

Si se examinan con detenimiento las actuales acciones previstas, se observa la amplitud del catálogo ya que, necesariamente, deben estar incluidas todas las políticas y medidas que responden a la tradicional clasificación. Con la reforma operada en la Ley de Empleo por el Real Decreto-ley 3/20116 se concretó, de modo más preciso, el espacio de actuación mediante la descripción legal de sus distintos ámbitos de interés (véase Figura 1).

FIGURA 1 - LÍNEAS DE ACTUACIÓN DE LAS POLÍTICAS ACTIVAS DE EMPLEO

\begin{tabular}{|c|l|}
\hline LínEAS DE ACTUACIón & \multicolumn{1}{c|}{ CONTENIDO DE LAS ACCIONES } \\
\hline Formación & $\begin{array}{l}\text { Programas destinados a reducir el abandono escolar. } \\
\text { Programas dirigidos a garantizar un mínimo de } \\
\text { formación inicial. } \\
\text { Institucionalización de requerimientos profesionales. } \\
\text { Desarrollo del reconocimiento de la formación. } \\
\text { Promoción de relaciones entre formación y empleo. }\end{array}$ \\
\hline $\begin{array}{c}\text { Creación directa de empleos y y } \\
\text { apoyo a nuevas iniciativas }\end{array}$ & $\begin{array}{l}\text { Iniciativas empresariales con carácter social. } \\
\text { Subvenciones a la contratación. } \\
\text { Iniciativas locales de empleo y yacimientos de empleo. } \\
\text { Desarrollo de nuevas modalidades de contratación. } \\
\text { Creación directa de empleo. } \\
\text { Medidas específicas dirigidas a la contratación de } \\
\text { jóvenes. } \\
\text { Reparto de empleo y reducción del tiempo de trabajo. }\end{array}$ \\
\hline $\begin{array}{c}\text { Desarrollo de sistemas de } \\
\text { ajuste oferta-demanda }\end{array}$ & $\begin{array}{l}\text { Asesoramiento y asistencia en la búsqueda de empleo. } \\
\text { Servicios públicos y privados de empleo. } \\
\text { Empresas de Trabajo Temporal. }\end{array}$ \\
\hline
\end{tabular}

FUENTE: elaboración propia a partir de Ruesga (2002).

Desde luego, el impacto de estas políticas vendría dado por la comprobación de que el nivel de inserción de los usuarios ha crecido significativamente, el tiempo transcurrido para la obtención del primer empleo se ha reducido considerablemente, su duración se ha elevado o se ha observado una mejora de salario y nivel de competencias adquirido (GARCÍA LÓPEZ, 2011; INSTITUTO NACIONAL DE EMPLEO, 2000). Al respecto, las posibilidades de inserción que ofrecen estas acciones influencian directamente en la predisposición para iniciarse en el terreno laboral.

6 Real Decreto-ley 3/2011, de 18 de febrero, de medidas urgentes para la mejora de la empleabilidad y la reforma de las políticas activas de empleo (BOE, 19/02/2011). 
En nuestro estudio, los graduados manifiestan sentirse poco preparados para emprender laboralmente (76\%), aun cuando diversos análisis han señalado la importancia de incidir sobre esta competencia. Por su parte, Ventura y Martínez (2004) sostienen que emprender y fomentar el espíritu de iniciativa deben convertirse en importantes preocupaciones de la educación superior, a fin de mejorar las posibilidades de empleo de los graduados, cada vez más llamados a crear puestos de trabajo y no solo a buscarlos. Y la Fundación Conocimiento y Desarrollo (2010), en su informe sobre los titulados universitarios y el mercado laboral, describe el espíritu emprendedor como el mayor déficit surgido entre las posibilidades existentes en el mercado de trabajo y la formación que poseen los graduados.

Con todo, no se trata de analizar la adecuación de la formación de estos titulados a su particular contexto de trabajo, sino de identificar aquellos elementos que dificultan su desarrollo profesional con el propósito de promover un análisis de mercado que constate la efectividad de las medidas actualmente activas. Los nuevos análisis presentados por el informe de la OCDE (2012) muestran un aumento en el gasto referido a políticas activas de empleo, a partir del comienzo de la crisis financiera, debido, probablemente, a la necesidad de mantener la orientación de la activación en tiempos de recesión. La potencialidad de las medidas, no obstante, se vio afectada por una disminución en los recursos destinados a las políticas activas, sobre todo en comparación con el incremento del número de demandantes en paro (ALUJAS, 2006; PALACIO; ÁLVAREZ, 2004). A pesar de que la proporción de recursos que España destina a políticas activas se encuentra en la media de los países de la UE, es un hecho que los datos referidos a nuestro país están lejos de mejorar con claridad en los últimos años (véase Figura 2).

De igual modo, las propias Administraciones Públicas apenas han analizado la influencia que estas iniciativas tienen en el acceso al empleo de los graduados, por más que en el plano internacional se haya extendido la tendencia a aceptar que su implementación en el mercado laboral implica, necesariamente, la evaluación de su impacto (ABDALA, 2004). No cabe olvidar que uno de los objetivos principales de las instituciones públicas pasa por transformar las políticas y mejorar su coordinación, además de ir implantando una cultura de la evaluación en esta materia (MINISTERIO DE EMPLEO Y SEGURIDAD SOCIAL, 2013).

En definitiva, el proceso de construcción de un Espacio Europeo de Educación Superior (EEES) no solo ha supuesto explorar una vía de «europeización», sino mostrar cómo las políticas sociales y educativas aparecen entrelazadas cada vez más con las políticas de empleo y con el mercado laboral (PEREYRA; LUZÓN; SEVILLA, 2006). Es así que el debate de las políticas públicas en 
FIGURA 2 - GASTO PÚBLICO* EN POLÍTICAS DE EMPLEO EN LA UE (Labour Market Policy Expenditure-LMP ${ }^{* *}$ )

\begin{tabular}{|l|c|c|c|c|c|c|c|c|}
\cline { 2 - 9 } \multicolumn{1}{c|}{} & $\mathbf{2 0 0 7}$ & $\mathbf{2 0 0 8}$ & $\mathbf{2 0 0 9}$ & $\mathbf{2 0 1 0}$ & $\mathbf{2 0 1 1}$ & $\mathbf{2 0 1 2}$ & $\mathbf{2 0 1 3}$ & $\mathbf{2 0 1 4}$ \\
\hline Unión Europea & 0,45 & 0,44 & 0,51 & 0,52 & 0,46 & - & - & - \\
\hline Bélgica & 0,47 & 0,51 & 0,54 & 0,56 & 0,64 & 0,59 & 0,52 & 0,52 \\
\hline Dinamarca & 0,98 & 0,94 & 1,13 & 1,36 & 1,38 & 1,31 & 1,29 & 1,40 \\
\hline Alemania & 0,55 & 0,55 & 0,62 & 0,52 & 0,43 & 0,34 & 0,30 & 0,29 \\
\hline Irlanda & 0,47 & 0,52 & 0,62 & 0,69 & 0,68 & 0,72 & 0,71 & 0,68 \\
\hline Grecia & 0,15 & 0,14 & 0,21 & 0,22 & - & - & 0,18 & 0,31 \\
\hline España & $\mathbf{0 , 6 1}$ & $\mathbf{0 , 5 9}$ & $\mathbf{0 , 6 4}$ & $\mathbf{0 , 7 1}$ & $\mathbf{0 , 6 9}$ & - & $\mathbf{0 , 4 2}$ & $\mathbf{0 , 4 5}$ \\
\hline Francia & 0,69 & 0,61 & 0,73 & 0,80 & 0,66 & 0,64 & 0,67 & 0,73 \\
\hline Italia & 0,36 & 0,36 & 0,35 & 0,32 & 0,30 & 0,34 & 0,32 & 0,28 \\
\hline Luxemburgo & 0,37 & 0,35 & 0,39 & 0,43 & 0,46 & 0,48 & 0,50 & 0,51 \\
\hline Hungría & 0,26 & 0,26 & 0,38 & 0,54 & 0,35 & 0,60 & 0,70 & 0,77 \\
\hline Países Bajos & 0,68 & 0,67 & 0,75 & 0,74 & 0,68 & 0,60 & 0,58 & 0,55 \\
\hline Austria & 0,49 & 0,50 & 0,64 & 0,64 & 0,55 & 0,55 & 0,59 & 0,62 \\
\hline Portugal & 0,36 & 0,39 & 0,61 & 0,54 & 0,44 & 0,38 & 0,46 & 0,53 \\
\hline Romania & 0,08 & 0,06 & 0,04 & 0,03 & 0,03 & 0,03 & 0,03 & 0,02 \\
\hline Finlandia & 0,68 & 0,64 & 0,71 & 0,83 & 0,82 & 0,84 & 0,86 & 0,91 \\
\hline Suecia & 0,77 & 0,64 & 0,67 & 0,84 & 0,91 & 1,01 & 1,07 & 1,08 \\
\hline Reino Unido & 0,04 & 0,04 & 0,05 & 0,08 & - & - & - & - \\
\hline Noruega & 0,43 & 0,41 & 0,47 & 0,49 & 0,44 & 0,41 & 0,38 & 0,38 \\
\hline
\end{tabular}

"Los datos corresponden al porcentaje de gasto público del Producto Interno Bruto (Percentage of gross domestic product).

${ }^{* *}$ Las categorías empleadas (2-7) del LMP responden a intervenciones que proporcionan apoyo temporal a grupos que presentan dificultades de acceso al mercado laboral y que se dirigen a reducir el desempleo, ayudar a los sujetos a pasar de una inactividad involuntaria al empleo o al mantenimiento de un puesto de trabajo. Categorías: formación, incentivos al empleo, apoyo al empleo y rehabilitación, creación directa de trabajo e incentivos al emprendimiento.

FUENTE: elaboración propia a partir de Eurostat (2016).

la educación superior ha despertado importantes reflexiones (CLARK, 1991), y una de ellas no puede situarse al margen del castigo al que se ha sometido a los graduados universitarios en cuestiones de empleo, sobre todo en cuanto a la consistencia entre trabajo al que se opta y formación académica que se posee (JIMÉNEZ VIVAS, 2009). El conjunto de los cambios técnicos y organizativos ha supuesto un cambio en la estructura de los empleos y de las calificaciones requeridas a los jóvenes que intentan acceder al mercado de trabajo (CACHÓN, 2004), configurando las políticas activas como el soporte principal de las acciones que deberían suponer una buena oportunidad para mejorar los procesos de inserción. No debe olvidarse que nos encontramos ante uno de los temas más 
controversiales a causa del carácter tan cambiante e inestable del mercado de trabajo, lo cual nos recuerda la necesidad de adaptar programas por objetivos a programas por competencias que favorezcan la inserción (SANTOS REGO, 2005).

\section{Conclusiones}

La transformación de la economía, la incorporación de la tecnología y las nuevas dinámicas del trabajo, junto con la aparición de nuevos productos y servicios, obligan a la sociedad en general y a las administraciones públicas, en particular, a tomar decisiones en sintonía con las necesidades que generan, a fin de avanzar hacia un desarrollo socioeducativo, económico y laboral equilibrado (INSTITUTO NACIONAL DE EMPLEO, 2000).

Hemos tratado de identificar las políticas activas de empleo como la pieza clave o, si se prefiere, el instrumento «estrella» de las medidas a adoptar. Pero estas han de proporcionar la oportunidad de mantener y mejorar la disponibilidad para el empleo de los graduados universitarios. Han de considerarse, además, como un instrumento para la mejora profesional y el aprendizaje de técnicas para la gestión de la carrera en situaciones caracterizadas por altas tasas de desempleo como las que existen hoy en día. Se debe recordar que se trata de medidas que favorecen el tránsito rápido del desempleo al empleo y de este a uno nuevo, pero también una herramienta para la mejora de la capacitación de las personas y de los puestos de trabajo de las empresas. Lo que cuenta, nos guste o no, es la competitividad de la economía.

Se necesita, por lo tanto, la reorientación de los programas de promoción del empleo con el fin de mejorar la eficiencia y eficacia de las políticas destinadas a fomentar la inserción laboral, dando prioridad a los jóvenes con el objetivo de reducir su elevada tasa de desempleo (MINISTERIO DE EMPLEO Y SEGURIDAD SOCIAL, 2013). Las políticas activas de empleo no solo constituyen el instrumento principal de las administraciones públicas, sino que en momentos de precariedad laboral son el único asidero de mejora. Es verdad que contamos con estudios que sitúan a los graduados dentro de los jóvenes con mayor inserción profesional (OBSERVATORIO DE LAS OCUPACIONES, 2014), pero también es una realidad que, en su mayoría, realizan actividades profesionales muy alejadas de su formación inicial.

La inserción laboral de los graduados universitarios, independientemente de su titulación, supone un desafío para la educación superior, en lo educativo y 
lo estrictamente profesional. Al respecto, la investigación ha de fijar dos puntos de análisis: la formación para la profesionalización y las posibilidades de acceso al mercado de trabajo. Lo que conviene es profundizar en el estudio de la realidad académica y de la educativa en el marco universitario. Debe haber una indagación específica de las competencias asociadas a trayectorias de potencial empleabilidad en una coyuntura histórica marcada por la incertidumbre y los apreciables niveles de precariedad laboral. En cualquier caso, la universidad sigue siendo el lugar adecuado para plantear tales cuestiones, toda vez que la calidad del currículo y las metodologías de aprendizaje en la educación superior continúen marcando diferencias.

\section{REFERENCIAS}

ABDALA, E. Manual para la evaluación de impacto en programas de formación para jóvenes. Montevideo: OIT, 2004.

ACSUG. Estudo da inserción laboral dos titulados no Sistema Universitario de Galicia 2009-2010. Santiago de Compostela: ACSUG, 2014.

ACUÑA, J. E. Modelo de gestión de calidad académica para la Educación Superior. Revista Calidad en la Educación Superior, 3, 1, 157-184, 2012.

ALONSO, L. E.; FERNÁNDEZ RODRÍGUEZ, C. J.; NYSSEN, J. M. El debate sobre las competencias. Una investigación cualitativa en torno a la educación superior y el mercado de trabajo en España. Madrid: ANECA, 2008.

ALUJAS, J. A. Las políticas activas de mercado de trabajo en España en el contexto de la Unión Europea. Revista del Ministerio de Trabajo y Asuntos Sociales, 61, 77-92, 2006.

ANECA. Los procesos de inserción laboral de los titulados universitarios en España. Factores de facilitación y de obstaculización. Madrid: ANECA, 2004.

BARBA, F. J. Políticas y servicios públicos de empleo. Una visión europea en conexión con el mundo universitario. Albacete: Bomarzo, 2012.

CACHÓN, L. Las políticas de transición: estrategia de actores y políticas de empleo juvenil en Europa. Estudios de Juventud, 65, 51-63, 2004.

CAJIDE, J. Calidad universitaria y empleo. Madrid: Dykinson, 2004.

CALIENDO, M.; SCHMIDL, R. Youth unemployment and active labor market policies in Europe. Journal of Labor Policy, 5, 1, 1-30, 2016. 
CLARK, B. El Sistema de Educación Superior. Una visión comparativa de la organización académica. México: Nueva Imagen, 1991.

COMISIÓN EUROPEA. El Fondo Social Europeo: Politicas Activas de Empleo y Servicios Públicos de Empleo. Bélgica: Comisión Europea, 2010.

EUROSTAT. LMP Expenditure. Bruselas, 2016. Disponible en: http://appsso.eurostat. ec.europa.eu/nui/onewindowpersession.do. Acceso en: 23 sept. 2016.

FERNÁNDEZ DOCAMPO, M. B. Funciones y disfunciones de la política nacional de empleo. In: CABEZA, J.; FERNÁNDEZ PROL, F. Políticas de empleo. Navarra: Aranzadi, 2013. 391-407.

FUNDACIÓN CONOCIMIENTO Y DESARROLLO. Graduados universitarios y mercado de trabajo. Barcelona: Fundación Conocimiento y Desarrollo, 2010.

GARCÍA LÓPEZ, J. R. Desempleo juvenil en España: causas y soluciones. Análisis Económico. Madrid: BBVA, 2011.

GOEDEGEBUURE, L.; KAISER, F.; MAASSEN, P.; MECK, L.; VAN VUGHT, F.; WEERT, E. Higher Education Policy. An International Comparative Perspective. Oxford: Pergamon Press, 1994.

HERNÁNDEZ-BEJARANO, M. La inserción de los jóvenes en el mercado laboral: un difícil reto para las políticas activas de empleo. In: CABEZA, J.; FERNÁNDEZ PROL, F. Políticas de empleo. Navarra: Aranzadi, 2013. 281-298.

INSTITUTO NACIONAL DE EMPLEO. Impacto de Politicas Activas de Empleo en los Universitarios. Madrid: Instituto Nacional de Empleo, 2000.

JIMÉNEZ VIVAS, A. Reflexiones sobre la necesidad de acercamiento entre universidad y mercado laboral. Revista Iberoamericana de Educación, 50, 1-8, 2009.

JOVER, D. Formación e inserción socio-laboral. Revista de Estudios de Juventud, 74, 95-112, 2006.

MINISTERIO DE EMPLEO Y SEGURIDAD SOCIAL. Estrategia de emprendimiento y empleo joven 2013/2016. Madrid: Ministerio de Empleo y Seguridad Social, 2013.

OBSERVATORIO DE LAS OCUPACIONES. Informe del Mercado de Trabajo de los Jóvenes. Madrid: Ministerio de Empleo y Seguridad Social, 2014.

OCDE. The OECD Job’s Study. Facts, Analysis, Strategies. París: OCDE, 1994.

OCDE. Perspectivas del empleo 2012. Informes OCDE. Madrid: Ministerio de Empleo y Seguridad Social, 2012.

OIT. Tendencias mundiales del empleo juvenil 2012. Resumen ejecutivo. Ginebra: OIT, 2012.

PALACIO, J. I.; ÁLVAREZ, C. El mercado de trabajo: análisis y políticas. Madrid: Akal, 2004. 
PEREYRA, M.; LUZÓN, A.; SEVILLA, D. Las universidades españolas y el proceso de construcción del Espacio Europeo de Educación Superior. Limitaciones y perspectivas de cambio. Revista Española de Educación Comparada, 12, 113-143, 2006.

ROCHA, F. Reflexiones y propuestas para la reforma de las políticas activas de empleo en España. Madrid: Centro Sindical de Estudios, 2010.

RODRÍGUEZ GÓMEZ, R. (2002). Continuidad y cambio de las políticas de educación superior. Revista mexicana de investigación educativa, 7, 14, 133-154, 2002.

ROMERO, V.; PÉREZ, E.; VIDAL, S.; JUEZ, M. Inserción Ocupacional. Barcelona: Altamar, 2004.

RUESGA, S. Economía del trabajo y política laboral. Madrid: Pirámide, 2002.

RUIZ DE MIGUEL, C.; GARCÍA DE LA BARRERA TRUJILLO, M. Inserción Laboral de los Licenciados en Pedagogía de la Universidad Complutense de Madrid (2006-2012): cómo, dónde y cuánto tardan en colocarse los pedagogos. Actas del XVI Congreso $\mathrm{Na}$ cional/II Internacional Modelos de Investigación Educativa de la Asociación Interuniversitaria de Investigación Pedagógica (AIDIPE). Alicante: AIDIPE, 2013. 1206-1213.

SÁIZ, J. M. Claves para un nuevo mercado de trabajo. Una aplicación a la Unión Europea. Alicante: Editorial Club Universitario, 2004.

SALVÀ, F. Trabajo, Formación y Ciudadanía. Conceptos básicos, políticas, programas y recursos. Madrid: Pirámide, 2009.

SANTOS REGO, M. A.; REQUEJO OSORIO, A.; RODRÍGUEZ MARTÍNEZ, A. Educación para la innovación y la competitividad. Formación y ocupación en el año 2000. Santiago de Compostela: Xunta de Galicia, 1993.

SANTOS REGO, M. A. La Universidad ante el proceso de convergencia europea: un desafío de calidad para la Unión. Revista Española de Pedagogía, 230, 5-16, 2005.

SANTOS REGO, M. A. Fines de la Universidad y Retos de la Empleabilidad en la Educación Superior. Procedia - Social and Behavioral Sciences, 16-25, 2014.

TÜNNERMANN, C. Pertinencia social y principios básicos para orientar el diseño de políticas de educación superior. Educación Superior y Sociedad, 11, 1, 181-196, 2000.

VENTURA, J.; MARTÍNEZ, F. Estudio sobre la inserción laboral de los graduados en Pedagogía en la Universidad de Barcelona. Barcelona: Universidad de Barcelona, 2004.

Texto recebido em 03 de noviembre de 2016. Texto aprovado em 15 de abril de 2017. 
